\title{
Steering and guiding light with light in a nanosuspension
}

\author{
Roland A. Terborg, ${ }^{1}$ Juan P. Torres, ${ }^{2,3}$ and Karen Volke-Sepulveda ${ }^{1,2, *}$ \\ ${ }^{1}$ Instituto de Fisica, UNAM, Apdo. Postal 20-364, 01000 Mexico D.F., Mexico \\ ${ }^{2}$ ICFO-Institut de Ciencies Fotoniques, 08860 Castelldefels, Barcelona, Spain \\ ${ }^{3}$ Department of Signal Theory and Communications, Univ. Politecnica de Catalunya, 08034 Barcelona, Spain \\ ${ }^{*}$ Corresponding author: karen@fisica.unam.mx
}

Received August 2, 2013; revised October 21, 2013; accepted November 8, 2013;

posted November 11, 2013 (Doc. ID 195202); published December 5, 2013

\begin{abstract}
We experimentally demonstrate guiding of a low-power probe beam (633 $\mathrm{nm}$ wavelength) by means of a lightinduced waveguide generated by the self-focusing of a strong pump beam (532 nm wavelength) in an artificial nonlinear medium, constituted by a colloidal suspension of dielectric nanoparticles. We also demonstrate optical steering of the probe beam by controlling the direction of propagation of the pump beam. The distance over which guiding is demonstrated $(5 \mathrm{~mm})$ is remarkably long when compared with previous experiments. (C) 2013 Optical Society of America

OCIS codes: (190.3970) Microparticle nonlinear optics; (230.7370) Waveguides; (190.6135) Spatial solitons; (140.7010) Laser trapping.

http://dx.doi.org/10.1364/OL.38.005284
\end{abstract}

In the early 1980s, it was demonstrated that a suspension of dielectric nanoparticles can act as an artificial nonlinear Kerr-like medium, which can be used for the generation and observation of bright optical spatial solitons (OSSs) [1] , degenerate four-wave mixing [2] , and for implementing bistable devices based on the self-focusing effect [3]. Qualitatively, the nonlinearity can be understood as a consequence of the existence of an optical gradient force, which attracts (repels) nanoparticles to (from) the regions of highest optical intensity when their refractive index $\left(n_{p}\right)$ is higher (lower) than that of the surrounding medium $\left(n_{m}\right)$. This leads to spatial variations of the effective refractive index directly related with the intensity distribution of the incident beam.

Although the phenomenon was initially described in terms of a pure Kerr nonlinearity [4]], it was recognized later that this assumption is an oversimplification. In fact, it is well known that a Kerr nonlinearity does not yield stable soliton propagation in the $(2+1) D$ case [5]. This fact renewed the interest on the subject and motivated the development of several theoretical models intended to elucidate the right kind of nonlinearity produced by the nanosuspensions. The general idea is to consider the medium as a gas of particles [ㅁ-ㅁ] , where diffusion is driven by the optical gradient force until reaching equilibrium conditions. This leads to diverse expressions for the position-dependent particle density $\rho(\mathbf{r})$, determined by the incident light spatial distribution and the characteristics of the nonlinear response of the medium.

In the slowly varying envelope approximation, the propagation of a light beam in the medium is described by

$$
i \frac{\partial \psi}{\partial z}+\frac{1}{2 k_{0} n_{b}} \nabla_{\perp}^{2} \psi+F(\rho) \psi=0
$$

where the optical field is given by $\mathbf{E}(\mathbf{r}, t)=\psi(\mathbf{r}) \exp$ $\left[i\left(k_{0} n_{b} z-\omega t\right)\right]$, with $k_{0}$ being the wavenumber of the light in vacuum, $\omega$ its frequency, and $n_{b}$ the refractive index of the medium in absence of light. $F(\rho)$ represents a complex function of the particle density (or concentration), whose real and imaginary parts are associated with the refractive index and Rayleigh scattering loss, respectively. The equation describing the interaction between the incident light and the medium, which is specified for each theoretical model, forms a coupled system with Eq. (1).

In order to explain the observation of stable propagation of OSS in $(2+1) D$, different considerations and assumptions have been made, including for example, the presence of nonlinear Rayleigh losses [7], hard-sphere potential interactions [8], or screened Coulomb repulsions ("van der Waals" gas) [9], which lead to different kinds of nonlinearities, from Kerr to exponential. With the aim of testing the different theoretical approaches, new experiments were conducted recently [10].

In this Letter, we present experimental results on the guiding of a weak probe beam (wavelength $\lambda_{r}=633 \mathrm{~nm}$ ) by means of the waveguide induced by an intense pump beam (wavelength $\lambda_{g}=532 \mathrm{~nm}$ ) in a nonlinear medium (NLM) constituted by a suspension of polystyrene nanoparticles. It is shown that the probe beam is not intense enough to induce a nonlinear effect by itself, but its transverse intensity profile is dramatically modified by the presence of the pump beam. Furthermore, we also demonstrate the capability to steer the probe beam in a range of over $4^{\circ}$ by controlling the incidence angle of the pump beam, with an essentially constant coupled power. Importantly, both beams propagate within the medium with a filament-like behavior for a distance of up to $5 \mathrm{~mm}$, which is considerably longer than distances reported in previous experiments, even in most of the theoretical works. In addition to the potential repercussions of these results for photonic engineering, experimental studies such as the one presented here pose new challenges for the theoretical understanding of the nonlinear response of nanocolloids.

A diagram of the experimental setup is shown in Fig. 1. A CW green laser $\left(\lambda_{g}=532 \mathrm{~nm}\right)$ with a Gaussian output mode and a red beam $\left(\lambda_{r}=633 \mathrm{~nm}\right)$ are used as the pump and probe beams, respectively. The propagation axis of the probe beam is set parallel to the pump beam by means of a beam combiner (BC1), so that both beams arrive parallel to the $20 \times$ microscope objective (MO). The 


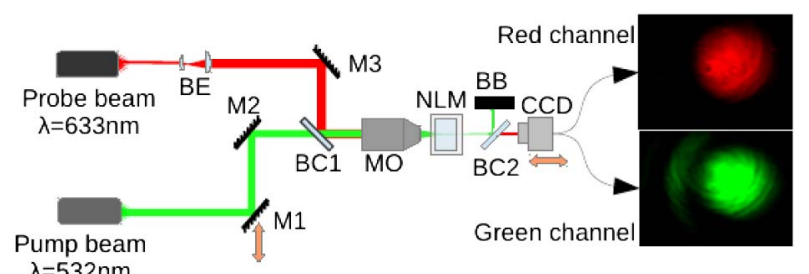

$\lambda=532 \mathrm{~nm}$

Fig. 1. Experimental setup. Beam expander (BE); mirrors (M1, M2 and M3); beam combiners (BC1 and BC2); microscope objective (MO); cell containing the nonlinear medium (NLM); beam block (BB); color CCD camera on a translation stage (CCD). Mirror M1 can be displaced to control the direction of propagation of the pump beam at the focal plane of MO.

pump beam can be displaced laterally by moving the mirror $\mathrm{M}_{1}$. When displaced, this beam passes through the same point at the focal plane, but its propagation axis is tilted with respect to the axis of the probe beam by a specific angle $\theta$. In principle, the maximum deviation angle would be limited by the maximum displacement of the pump beam that can be achieved while still fitting complete into the aperture of the microscope objective, which is of approximately $\pm 6^{\circ}$, giving a range of up to $12^{\circ}$. Nevertheless, the field of view of our detection system only allows a reliable quantitative characterization of the beam profile and deviation angle within a limited range of up to $4^{\circ}$. Qualitatively, we were able to observe deviation angles of about $\pm 3^{\circ}$ without significant changes in the beam profile. We optimize the coupling of the probe beam to the waveguide generated by the pump beam by matching the spot size of both beams using a beam expander (BE).

The NLM consists of a suspension of polystyrene nanospheres of $50 \mathrm{~nm}$ diameter with refractive index $n_{p}=$ $1.60\left(n_{p}=1.59\right)$ at $532 \mathrm{~nm}(633 \mathrm{~nm})$. The nanoparticles are immersed in distilled water, with a concentration of $1 \%$ solids, giving a scattering loss of $\alpha_{0} \sim 3.4 \mathrm{~cm}^{-1}$. The focal plane is located inside the cuvette containing the suspension, at a distance of $\sim 3 z_{R}$ from the wall, where $z_{R} \approx 18 \mu \mathrm{m}$ denotes the Rayleigh range of the pump beam in water. The length of the cuvette along the propagation axes of the beams is $5 \mathrm{~mm}$, with glass walls of approximately $1 \mathrm{~mm}$ width. Another dichroic mirror (BC2) is used to remove most of the pump beam emerging from the NLM to avoid saturation of the detection system. The transverse profiles of the probe beam and the remaining of the pump beam are imaged onto a CCD camera at different distances along the propagation axis within a relatively reduced range $(\sim 12.7 \mathrm{~mm})$, since they become divergent when emerging from the sample cuvette. In this way we are able to simultaneously observe both beams, which are then digitally separated in green and red channels. While the total output power of the probe beam is kept constant $(<25 \mathrm{~mW})$, the power of the pump beam is varied up to $1500 \mathrm{~mW}$. Experiments were carried out for different pump powers and deviation angles $\theta$.

First, we look for the power necessary to start observing nonlinear effects, i.e., self-focusing. The beam starts to shrink gradually as the power is increased, and the self-focusing effect becomes noticeable for powers $\gtrsim 600 \mathrm{~mW}$. Hence the probe beam, whose power is well below this threshold, cannot induce any nonlinear effects by itself. This can be clearly observed in Fig. 2, which shows images of both beams at the output of the sample. The top row of Fig. 2 shows the pump beam for two different values of the optical power (939 and $1266 \mathrm{~mW}$ ). The second row shows the shape of the probe beam when the pump beam is switched off. The bottom row shows the probe beam under the influence of the copropagating pump beam. The intensity distribution of the probe beam is dramatically modified by the presence of the pump.

The change in the spatial structure of the probe seems to be directly related to the structure of the pump beam. When the pump power is increased over $\gtrsim 900 \mathrm{~mW}$, some noticeable changes of its spatial structure take place. The input beam profile, showing a Gaussian-like structure with a single maximum at the center, changes to exhibit a bright core, containing most of the energy, surrounded by portions of concentric bright rings on one side. The number, geometry, and spacing of the rings vary with the power. In these circumstances, the probe beam also resembles the profile of the pump. The presence of this outer structure becomes more evident as the power increases and its uneven shape seems to indicate a symmetry breaking, possibly related to a convective flow that in turn modifies the nanoparticle concentration.

When the axis of the focused pump beam is tilted by displacing the mirror M1, a corresponding deviation in the probe beam is observed. Figure 3 illustrates how the probe beam is deflected to different angles. In our current experimental setup, the probe beam exhibits no significant changes in shape and size as the pump beam is tilted within the whole available range of approximately $4^{\circ}$, suggesting that larger tilting angles could be achieved
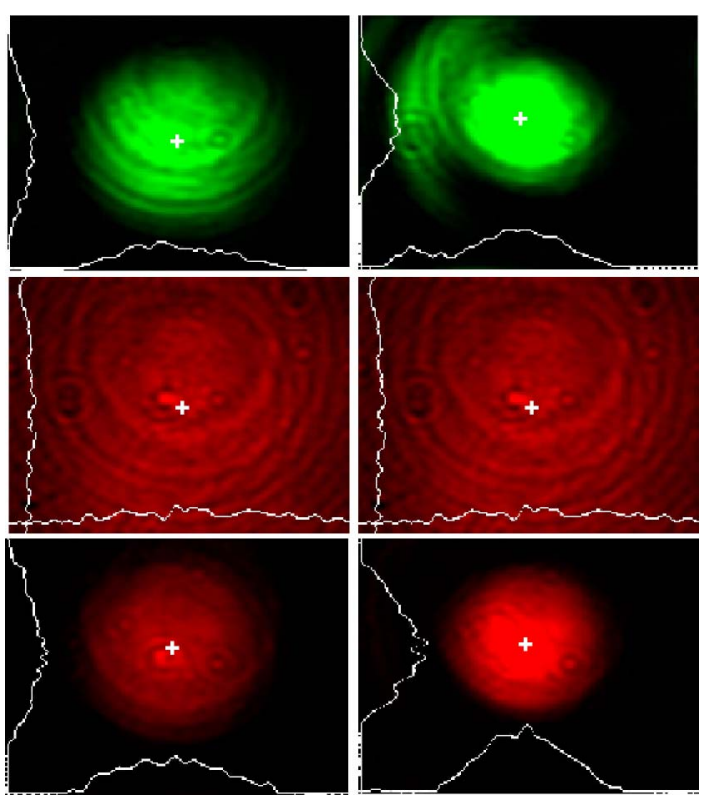

Fig. 2. Images of the output pump beam (top row) and of the probe beam when the pump is off (middle row) and on (bottom row). Left (right) column corresponds to a pump power of $939 \mathrm{~mW}(1266 \mathrm{~mW})$. A white-cross marker indicates the intensity centroid in each case. The white curves show vertical and horizontal intensity profiles in each image. The intensity in the images of the central row was multiplied by a factor of 3 to make them visible. 


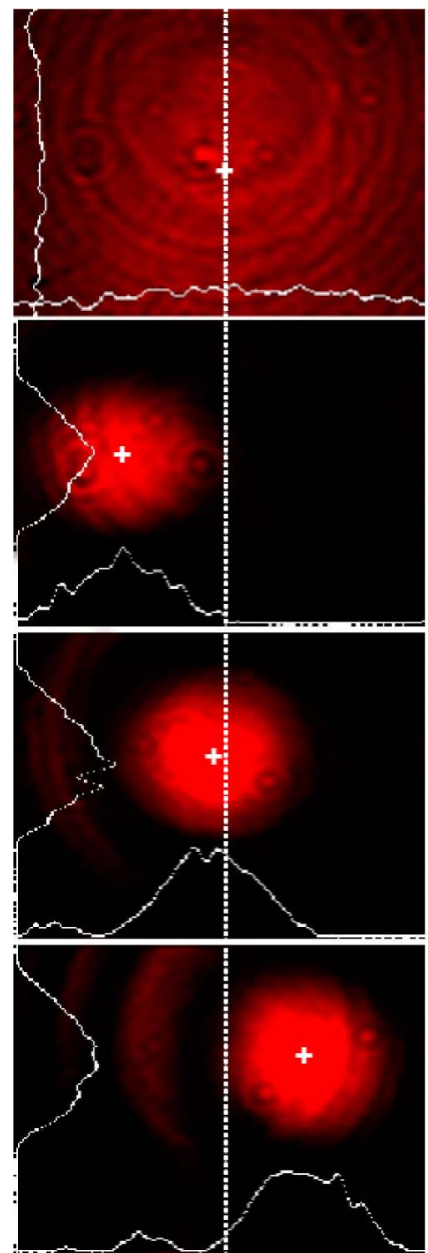

Fig. 3. From top to bottom: probe beam in absence of the pump beam; probe beam guided by the pump beam propagating at angles of deflection of $-1.8^{\circ}, 0^{\circ}$, and $1.8^{\circ}$, respectively, for a pump power of $1434 \mathrm{~mW}$. The centroid in each case is indicated with a white-cross, and the white curves show vertical and horizontal intensity profiles. The dotted line shows the position of the centroid of the nonguided probe beam. The intensity in the top image was multiplied by a factor of 3 to make it visible.

with a more flexible setup. Figure $\underline{4}$ illustrates what we have called the confined power factor, which is the ratio of the power in the guided probe beam to the power in the nonguided probe beam (when the pump is off) in a fixed area. The measured power corresponds to the intensity integrated over a circular aperture of fixed radius, given by the value where the intensity in the guided probe has decreased to 0.25 of its maximum value for $\theta=0$. The confined probe power increases with the pump power, as shown by inspecting the different curves in Fig. 4 , going from over 2 for $939 \mathrm{~mW}$ of pump power to up to 5 for $1434 \mathrm{~mW}$. However, the confined power factor remains essentially constant as a function of the deviation angle for each pump power, with small variations attributed to inhomogeneities in the intensity profile of the nonguided probe beam within the integration area, as well as to a small experimental uncertainty in the position of the centroid of the guided beam, used as the center of the aperture.

Some of the experimental results reported here might help unveil the detailed structure of the nonlinearity

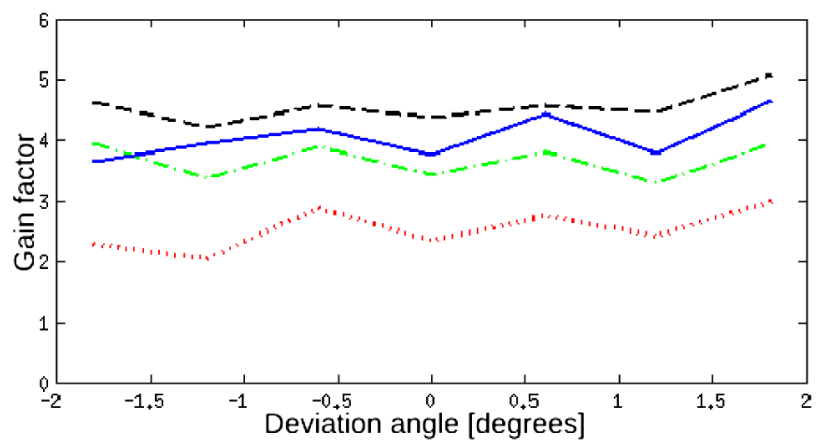

Fig. 4. Power gain of the guided probe beam when compared with the nonguided probe beam, for an aperture of fixed radius, as a function of angle of deviation for different pump powers. Dotted line (red): $939 \mathrm{~mW}$; dash-dot line (green): $1123 \mathrm{~mW}$; solid line (blue): $1266 \mathrm{~mW}$; dashed line (black): $1434 \mathrm{~mW}$.

induced by the nanosuspension. The main reason that permits these new observations is that the self-focused pump beam is allowed to propagate a considerably longer distance (at least one-order of magnitude longer) than previous experiments of the same kind $[1,3,10]$. It even exceeds distances reported in theoretical studies [ $[-9]$. One especially relevant observation is the appearance of an annular structure around the main core of the self-trapped beam. In a recent study [11], it was shown that high-power self-trapped beams can shed energy away due to long-lived oscillations in amplitude and width, caused by periodic focusing and defocusing. This could explain the formation of the ring structure we observe in our experiments. The propagation distances required for this observation are consistent with those in our experiments.

Finally, it is worth mentioning that a recent work has reported the self-trapping of an optical beam in a nanosuspension due to the thermophoretic effect [12]. Although we cannot rule out the possible influence of thermal effects in our experimental results, there are important aspects that lead us to attribute our results to the presence of optical gradient forces in the first instance. Namely, the thermophoretic transport is characterized by the Soret coefficient $\boldsymbol{S}_{T}$, which is the ratio between the thermophoretic mobility and the Brownian diffusion coefficient. If $S_{T}>0\left(S_{T}<0\right)$ the particles are thermophobic (thermophillic), moving from hotter (colder) to colder (hotter) regions. According to [13], $S_{T}=0$ for polystyrene nanospheres of $25 \mathrm{~nm}$ radius at $T \sim 26^{\circ} \mathrm{C}$, as our particles. At room temperature $\left(20^{\circ} \mathrm{C}<T<24^{\circ} \mathrm{C}\right)$, the Soret coefficient is considerably smaller than the corresponding value for nanoparticles of $10 \mathrm{~nm}$-radius, as those used in [12]. On the other hand, in [12] the authors use a NIR laser at $808 \mathrm{~nm}$, for which water absorption is almost two orders of magnitude larger than for the green light used in our experiment, and thus thermal gradients are expected to be much stronger in that case. Finally, the conditions to obtain negative thermophoresis, as it is required for a self-focusing nonlinearity, imply a high degree of control on the solvent properties and particlesolvent interface [12-14], which we do not have in our experiment.

In conclusion, we have demonstrated optical guiding and steering of a low-power $(<25 \mathrm{~mW})$ probe beam by 
means of a light-induced waveguide generated by a pump beam propagating through a colloidal medium, due to the action of optical trapping forces. The stable propagation of both beams along distances of up to $5 \mathrm{~mm}$ was observed. By steering the pump beam, the probe beam can be deviated within a range of over $4^{\circ}$. The behaviors of both the pump and the probe beam are insensitive to polarization, in contrast to the case of optical solitons induced in liquid crystals [15]. The ability of controlling light with light in soft matter in the way demonstrated here may be extended with the use of optically addressed versatile systems [16]. From a fundamental viewpoint, our results raise new questions about the nature and the physical origin of the nonlinearity in nanosuspensions.

This work was partially supported by CONACYT Mexico, project 132527. R. T. acknowledges support from CONACYT for the grant 323560. K. V.-S. also acknowledges support from CONACYT (grant 186368) and DGAPA-UNAM for the sabbatical grants. We acknowledge interesting discussion with professor Y. Kartashov.

\section{References}

1. A. Ashkin, J. M. Dziedzic, and P. W. Smith, Opt. Lett. 7, 276 (1982).
2. P. W. Smith, A. Ashkin, and W. J. Tomlinson, Opt. Lett. 6, 284 (1981).

3. P. W. Smith, A. Ashkin, J. E. Biorkholm, and D. J. Eilenberger, Opt. Lett. 9, 131 (1984).

4. P. W. Smith, P. J. Maloney, and A. Ashkin, Opt. Lett. 7, 347 (1982).

5. G. I. Stegeman and M. Segev, Science 286, 1518 (1999).

6. R. Gordon and J. T. Blakely, Phys. Rev. A 75, 055801 (2007).

7. R. El-Ganainy, D. N. Christodoulides, C. Rotschild, and M. Segev, Opt. Express 15, 10207 (2007).

8. M. Matuszewski, W. Krolikowski, and Y. S. Kivshar, Opt. Express 16, 1371 (2008).

9. R. El-Ganainy, D. N. Christodoulides, E. M. Wright, W. M. Lee, and K. Dholakia, Phys. Rev. A 80, 053805 (2009).

10. W. M. Lee, R. El-Ganainy, D. N. Christodoulides, K. Dholakia, and E. M. Wright, Opt. Express 17, 10277 (2009).

11. C. Travis, G. Norris, G. McConnell, and G.-L. Oppo, Opt. Express 21, 23459 (2013).

12. Y. Lamhot, A. Barak, O. Peleg, and M. Segev, Phys. Rev. Lett. 105, 163906 (2010).

13. M. Braibanti, D. Vigolo, and R. Piazza, Phys. Rev. Lett. 100, 108303 (2008).

14. S. A. Putnam and D. G. Cahill, Langmuir 21, 5317 (2005).

15. M. Peccianti, C. Conti, G. Assanto, A. De Luca, and C. Umeton, Nature 432, 733 (2004).

16. T. Čižmár, O. Brzobohatý, K. Dholakia, and P. Zemánek, Laser Phys. Lett. 8, 50 (2011). 